\title{
Adenylate Energy Charge-New Tool for Determining Metalworking Fluid Microbial Population's Sublethal Response to Microbicide Treatment
}

\author{
Frederick J Passman ${ }^{1}{ }^{*}$, Peter Küenzi ${ }^{2}$ and Jordan Schmidt ${ }^{3}$ \\ ${ }^{1}$ Biodeterioration Control Associates, New Jersey, USA \\ ${ }^{2}$ Blaser Swisslube, USA \\ ${ }^{3}$ LuminUltra Technologies, Canada
}

*Corresponding author: Frederick J Passman, Biodeterioration Control Associates, Inc., PO Box 3659 Princeton, New Jersey 085433659, USA.

To Cite This Article: FrederickJ Passman, Adenylate Energy Charge-New Tool for Determining Metalworking Fluid Microbial Population's Sublethal Response to Microbicide Treatment. 2020 - 7(4). AJBSR.MS.ID.001178. DOI: 10.34297/AJBSR.2020.07.001178.

Received: 制 February 3, 2020; Published: 㘹 February 26, 2020

\begin{abstract}
Adenylate energy charge (AEC) is computed from the ratios of three energy molecules found in all living cells: adenosine triphosphate (ATP), adenosine diphosphate (ADP), and adenosine monophosphate (AMP). Previous studies have shown that when microbicide treatments resulting in ATP concentration decreases of $<2 \log 10 \mathrm{pg}^{\mathrm{ATP}} \mathrm{mL}^{-1}$, populations recover within a few days post-treatment. Recovery has not been observed when microbicide treatments reduce ATP-bioburdens by $\geq 2 \log _{10}$ pg ATP mL- 1 . However, frequently $\geq 1 \log _{10}$ pg ATP mL ${ }^{-1}$ remains even after effective treatment. The AECs of robust microbial communities range from 0.7 to 0.95 . When populations are stressed, the AEC decreases - reflecting the relative depletion of ATP and accumulation of ADP and AMP within cells. The paper reports the impact of lethal and sub-lethal microbicide treatments on AEC in microbially contaminated emulsifiable oil and semi-synthetic metalworking fluids. The results demonstrate the utility of AEC testing to determine the physiological state of microbial contaminants in water-miscible metalworking fluids. This capability is becoming increasingly important as metalworking fluid move from microbicide use to reliance on bio resistant functional additives.
\end{abstract}

Keywords: Adenylate Energy Charge, AEC, Adenosine Triphosphate, ATP, Bacterial, Bioburden, Biocides, Metalworking Fluids, Microbial Contamination, Microbicides, MWF

Abbreviations: AEC: adenylate energy charge; ADP: adenosine diphosphate; AMP: adenosine monophosphate; AP: amine package (proprietary); ATP: adenosine triphosphate; AXP: combination of ADP + AMP + ATP; BIT - 1,2-benzisothiazol-3(2H)-one; cADP, cAMP, cATP: cellular adenosine di-, mono-, and triphosphate, respectively; EO: emulsifiable oil; F-C: flow cytometry; HCHO: formaldehyde; MBO: 3,3'-methylenebis [5-methyloxazolidine]; MWF: metalworking fluid; RLU: relative light unit; TAE: Technische Akademie Esslingen

\section{Introduction}

Water-miscible metalworking fluids (MWFs) are used to provide cooling, lubrication, and waste (metal fines) transport in machining and metal forming operations. Typically, MWFs are concentrated blends of functional additives in a base stock. When the base stock is petroleum, animal, or vegetable oil, the MWF is classified as an emulsifiable oil [1]. For end-use, MWF are diluted to $3 \%$ (vol) to $10 \%$ (vol) in water. End-use diluted MWF are recirculated at high velocities $\left({ }^{3} 3 \mathrm{~m} 3 \mathrm{~min}^{-1}\right)$, under turbulent-flow conditions. This well-aerated, aqueous-organic mixture, operating at $25^{\circ} \mathrm{C}$ to $35{ }^{\circ} \mathrm{C}$ creates optimal conditions for microbial growth and proliferation [2]. Abundant microbial growth in recirculating MWFs and on MWF system surfaces are reservoirs for bioaerosols in machining facilities. Bioaerosols can cause respiratory diseases ranging from mild allergic rhinitis to lethal hypersensitivity pneumonitis [3]. Consequently, effective microbial contamination control measures are necessary to prevent both MWF biodeterioration and worker health risks. Historically, antimicrobial pesticides (also known as microbicides, or biocides) have been used in formulation, 
added tank side, or both, to control bioburdens in MWF. However, recent regulatory trends have restricted MWF microbicide use [4]. In response, MWF formulators have turned to bio resistant performance additives. These additives are not intended to be microbicidal. However, they render the MWF less accommodating to microbial contamination [5]. To properly assess the relationship between bio resistant additives and bioburdens in MWFs, new tools are needed. The use of adenosine triphosphate (ATP) testing by ASTM method E2694 [6] was discussed at previous TAE Tribology colloquia $[7,8]$. Atkinson and Walton reported in 1966 [9], the ratios of the adenosine nucleotides: ATP, ADP, and AMP, provided an index of the population's physiological state, as described by the equation:

$$
\mathrm{AEC}=([\mathrm{cATP}]+0.5[\mathrm{cADP}]),([\mathrm{cATP}]+[\mathrm{cADP}]+\mathrm{cAMP}]) .
$$

Where AEC was the adenylate energy charge, and [CATP], [cADP], and [CAMP] were the respective concentrations of cellular ATP, ADP and AMP in pg $\mathrm{mL}^{-1}$. The AEC of healthy, metabolically active microbial populations was typically ${ }^{3} 0.78$ [10]. Stressed but viable populations typically had AECs in the 0.44 to 0.78 range and moribund populations had AECs < 0.44 [10]. In the 1970s there were several reports of AEC profiles for natural populations $[11,12]$ This paper reports the impact of biostable amine additives and of selective microbicides on the AEC of metalworking fluid (MWF) microbial populations.

\section{Materials and Methods}

\section{AXP testing}

Adenosine nucleotides were extracted from MWF samples by ASTM Method E2694 [6]. Briefly, a $5.0 \mathrm{~mL}$ specimen of MWF was filtered through a $0.7 \mathrm{~mm}$, in-line glass fiber filter. The retentate was washed with a propriety rinse solution to eliminate interferences and then retained cells were lysed, with the lysate pressure filtered into a dilution buffer. The extract was then reacted with a Luciferin-Luciferase substrate-enzyme, and luminescence was measured using a luminometer. Raw relative light unit (RLU) results were compared against ATP, ADP, and AMP reference standards to yield quantitative results in $\mathrm{pg} \mathrm{mL}^{-1}$ of each adenosine nucleotide. The AEC was computed using equation [1].

\section{Results}

\section{Precision evaluation}

Table 1: Repeatability precision of AXP test parameters in EO MWF.

\begin{tabular}{|c|c|c|}
\hline Parameter & s & CV \% \\
\hline Log10[cATP] & 0.078 & 2.4 \\
\hline Log10[cADP] & 0.17 & 0.5 \\
\hline Log10[cAMP] & 0.077 & 2.6 \\
\hline AEC & 0.036 & 5.1 \\
\hline
\end{tabular}

The AXP method's precision was evaluated by treating an EO MWF with BIT at either 600 ppmv (a.s.) or 2500 ppmv (a.s.) and

\section{Culture testing}

The standard plate count (spread plate) method was used to enumerate culturable bacteria.

\section{Flow cytometry}

Bacterial cells were isolated by gradient centrifugation $(10.5 \%$ Nycodenz) and re-suspended in a Tris-EDTA buffered system $(\mathrm{pH}$ 8.3), stained with SybrGreen $(1: 100,000)$ and subsequently analyzed on an Accuri C6 cytometer (BD Biosciences).

\section{MWFs and test substances}

Testing was performed on proprietary emuslifiable oil (EO) MWF formulations diluted to $5 \%$ (vol) in laboratory tap water. Depending on the individual experiment, dilute MWF was dosed with: 1,2-benzisothiazol-3(2H)-one (BIT, $20 \%$ (vol) active ingredient - a.i. - as supplied - a.s.)) at either 600 ppmv or 2,500 ppmv; formaldehyde (HCHO) at $100 \mathrm{ppmv}, 370 \mathrm{ppmv}$, or 3,700 ppmv (all a.s.); 3,3'-methylenebis[5-methyloxazolidine] (MBO, 99\% a.i.) at 250 ppmv, 500 ppmv, 1,000 ppmv, or 2,000 ppmv (all a.s.), two proprietary amine additive packages (AP1 and AP2). The total amine concentration in AP1 was 59. \% vol. (1.19\% vol in diluted MWF) and that of 11900 ppm AP2 was $68.0 \%$ vol. (1.36 \% vol. in diluted MWF).

\section{Testing}

Uncharacterized microbial inocula from contaminated MWFs were grown in untreated, end-use diluted MWFs until ATP-bioburden ([cATP]) was ${ }^{3} 4 \log _{10}$ pg $\mathrm{mL}^{-1}$. The high bioburden MWF was then dispensed into the appropriate number of test jars and treated with a test substance. Specimens were then collected periodically-the number of sampling times and sampling intervals varying among experiments - and tested by AXP. During some experiments, AXP testing was augmented with culture testing, flow-cytometry counting, or both.

\section{Statistics}

All statistical testing was performed using Microsoft Excel's data analysis add-in software. sampling after 24h (T24) and 96h (T96). Triplicate specimens were tested at each time point to give a total of 15 specimens. Average 
standard deviations (s) and coefficients of variation (CV \%) are shown in Table 1.

\section{Relationship between [cATP], AEC, culturability, and flow cytometry cell counts.}

In the first experiment, eight MWF samples were collected and tested by AXP and culturability. Based on the minimal variability determined for AEC results, replicate tests were not performed for this or subsequent tests. The [CATP] and CFU $\mathrm{mL}^{-1}$ results were transformed to attribute scores (negligible bioburden $=1$, moderate bioburden $=3$, and heavy bioburden $=5$ ). Table 2 shows that for all samples, ATP and culture-based attribute scores agreed and that - except for sample 8 - when bioburdens were negligible, AEC $<0.4$. Sample 8 differed from the other low bioburden MWFs in that bioburdens were at the high end of the negligible range $\left(\log _{10}[-\right.$ cATP] $<2$ pg $\mathrm{mL}^{-1} ; \log _{10} \mathrm{CFU} \mathrm{mL}^{-1}<3$ ). Although the bioburden's attribute score was negligible the microbes present were metabolically active. In a second experiment a Bio concept EO MWF was treated with $\mathrm{HCHO}$ at 3,700 ppmv. Samples were taken a $\mathrm{T}_{0}$, $\mathrm{T}_{30} \mathrm{~min}, \mathrm{~T}_{60}$ min, and $\mathrm{T}_{120} \mathrm{~min}$, and tested for AEC, culturability and direct counts using flow cytometry. Culturability at $\mathrm{T}_{0}$ was $6 \log _{10}$ CFU mL ${ }^{-1}$ and BDL (below detection limits; $<1 \log _{10} \mathrm{CFU} \mathrm{mL}{ }^{-1}$ ) at all other sampling times. As shown in Figure 1, HCHO treatment had no measurable effect on flow-cytometry counts. Given that HCHO is used as a preservative to prevent cells from lysing, this is not surprising. Within $2 \mathrm{~h}$, the AEC fell from 0.92 to 0.40 - demonstrating that the population had shifted from active metabolism to a moribund state. The [cAMP] increase mirrored the [cATP] decrease.

Table 2: Comparison of $\log _{10}[\mathrm{CATP}]$ and $\log _{10} \mathrm{CFU} \mathrm{mL}^{-1}$ data from eight MWF samples.

\begin{tabular}{|c|c|c|c|c|c|c|}
\hline \multirow{2}{*}{ Sample } & \multirow{2}{*}{ Product } & \multirow{2}{*}{$\log _{10}$ [CATP] } & \multirow{2}{*}{$\mathrm{CFU} \mathrm{mL}^{-1}$} & \multirow{2}{*}{ AEC } & \multicolumn{2}{|c|}{ Attribute Scores } \\
\hline & & & & & [cATP] & CFU \\
\hline 1 & $\mathrm{EO}+$ Biocide $^{\mathrm{a}}$ & 0.96 & $(0)$ & 0.1 & 1 & 1 \\
\hline 2 & $\mathrm{EO}-\mathrm{BC}^{\mathrm{b}}$ & 4.35 & 7.37 & 0.83 & 5 & 5 \\
\hline 3 & EO-BC & 3.64 & 6.89 & 0.83 & 5 & 5 \\
\hline 4 & E01 & 1 & $(0)$ & 0.12 & 1 & 1 \\
\hline 5 & E02 & 0.72 & $(0)$ & 0.16 & 1 & 1 \\
\hline 6 & E03 & 0.75 & $(0)$ & 0.04 & 1 & 1 \\
\hline 7 & E04 & 0.73 & $(0)$ & 0.16 & 1 & 1 \\
\hline 8 & E05 & 1.52 & 2.6 & 0.82 & 1 & 1 \\
\hline
\end{tabular}

Notes:

a EO MWF had been formulated with unidentified microbicide.

b BC - Bio concept formulation - known to selectively support Pseudomonas oleo orans proliferation.

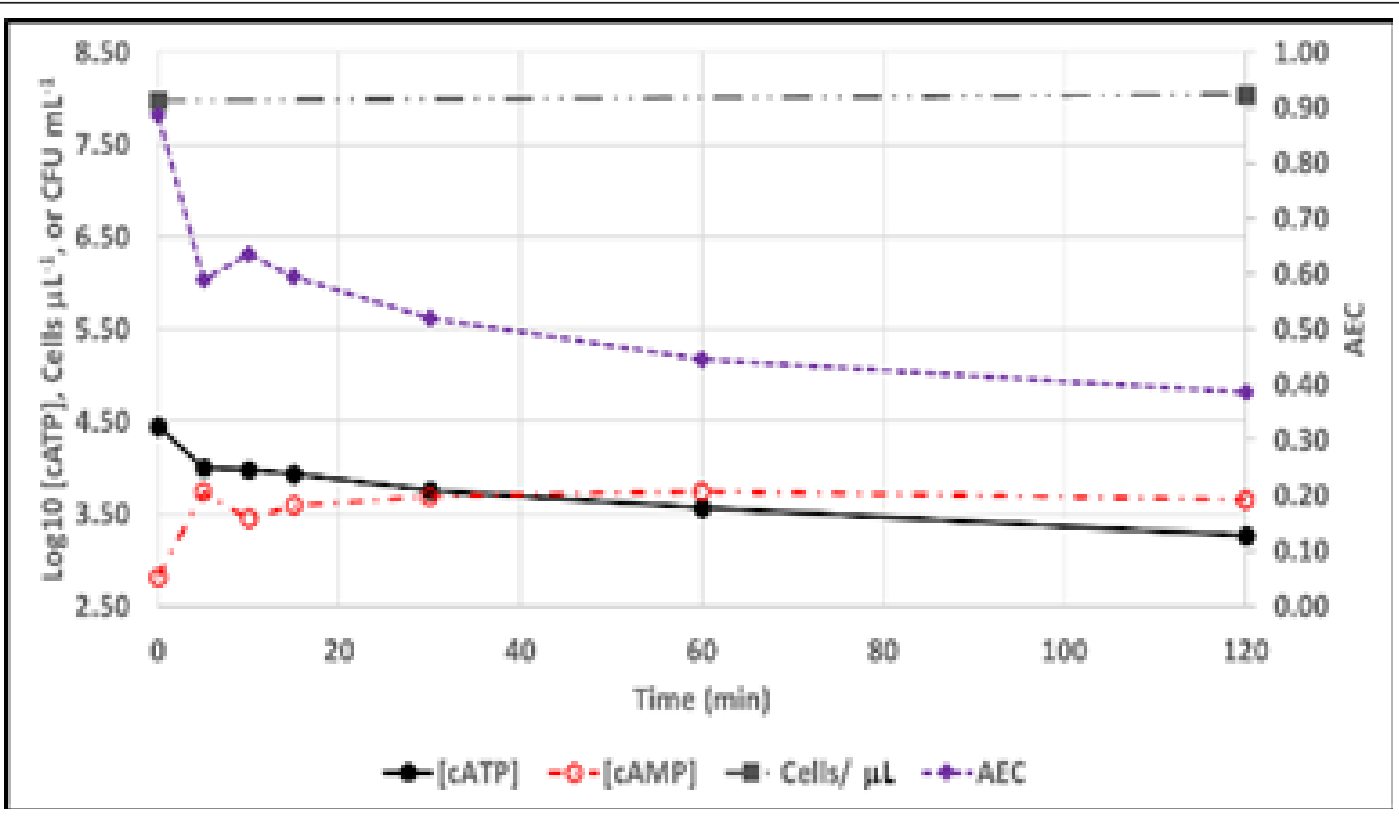

Figure 1: Effect of 3,700 ppmv HCHO v. population metrics in $5 \%$ Bio-Concept EO MWF. 
Microbicide performance is invariably MWF-formulation dependent. As shown in Table 3, BIT at 2500 ppmv (a.s.) was ineffective in the emulsifiable MWF used for this evaluation. In this test series, $\mathrm{D}[\mathrm{cATP}]$ max was $<2$ for all treatments and times. Moreover, at $264 \mathrm{~h}$, both [cATP] and CFU mL ${ }^{-1}$ had increased from their $168 \mathrm{~h}$ values. Neither the AEC nor flow-cytometry (F-C) count values in treated MWF were substantially different from those in the control. The AEC values indicated that the population remained stressed but viable in both the control and treated MWFs.

Table 3: Effect of BIT treatment on MWF bioburden.

\begin{tabular}{|c|c|c|c|c|c|c|}
\hline Treatment & Time (h) & $\log _{10}[\mathrm{cATP}]$ & $\Delta \log _{10}[$ сАТП] & AEC & $\log _{10}$ CFU mL $\mathrm{m}^{-1}$ & F-C Counts \\
\hline Control b & 168 & 3.93 & 0 & 0.51 & 7.96 & 9.54 \\
\hline $0.06 \%$ BIT & 168 & 3.41 & 0.52 & 0.55 & 7 & 8.79 \\
\hline $0.06 \% \mathrm{BIT}$ & 264 & 3.56 & 0.37 & 0.54 & 7.64 & 8.74 \\
\hline $0.25 \%$ BIT & 168 & 2.27 & 1.66 & 0.51 & 1.78 & 8.75 \\
\hline $0.25 \% \mathrm{BIT}$ & 264 & 2.92 & 1.01 & 0.45 & 4.7 & 8.81 \\
\hline
\end{tabular}

Notes:

a [cATP] are pg mL-1; F-C counts are counts $\mathrm{mL}-1$.

b Emulsifiable oil MWF.

Table 4: Effect of non-biocidal, amine additives on MWF bioburden.

\begin{tabular}{|c|c|c|c|c|}
\hline Treatment & $\log _{10}[\mathrm{cATP}]$ & $\log _{10}$ CFU mL $\mathrm{mL}^{-1}$ & $\log _{10}$ Cells $\mathrm{mL}^{-1}$ & AEC \\
\hline \multicolumn{5}{|l|}{ MWF 34928} \\
\hline Control & 3.75 & 6.62 & 6.64 & 0.87 \\
\hline Amine 1 & 2.24 & 5.68 & 6.6 & 0.39 \\
\hline Amine 2 & 2.24 & 5.72 & 6.26 & 0.41 \\
\hline \multicolumn{5}{|l|}{ MWF 34929} \\
\hline Control & 4.11 & 6.7 & 6.97 & 0.92 \\
\hline Amine 1 & 2.35 & 5.96 & 6.42 & 0.33 \\
\hline Amine 2 & 2.2 & 5.54 & 6.86 & 0.22 \\
\hline
\end{tabular}

Two emulsifiable oil MWF were augmented with two amine additive packages, and tested for $\log _{10}$ [cATP], AEC, $\log _{10}$ CFU mL $\mathrm{mL}^{-1}$ and $\log _{10}$ F-C count at time $0,24 \mathrm{~h}, 48$, and $72 \mathrm{~h}$. The results, shown in Table 4 indicate that [CATP] and AEC were affected, but culturability and cell counts were not. These results indicated that the amine packages had a biostatic effect - stressing but not killing the contaminant microbes.

\section{Discussion}

Routine MWF microbial contamination testing by ASTM E2694 [6] has proven to be a useful, culture-independent tool. A modified version of the ATP test differentiates between bacterial and fungal contamination [13]. Although ATP-bioburdens and culture test recoveries often correlate well [14] there are occasions when disagreements between the two parameters occur. Two conditions can contribute to these disagreements. When viable microbes do not proliferate on growth media, culture test results will underestimate the population density [14]. Conversely, in biostatic MWFs, moribund or dormant cells can recover once they have been transferred from the MWF to a nutrient medium. Environmental pressures adverse to microbial communities can be reflected in AEC values [10]. The data presented in this paper show that when culture and flow-cytometry testing indicate a greater bioburden than that indicated by ATP, AEC are consistently <0.5. Consequently, AEC appears to be a useful parameter for assessing the overall physiological state of microbial populations in MWFs. This is unlikely to be useful for routine testing but might be a valuable tool for evaluating biostatic MWF formulations and those augmented with adjuvants.

\section{Conclusions}

Determination of [cATP], [cADP], and [cAMP] from which to compute AEC provides consistent and useful insights into the metabolic state of microbial populations in water miscible MWFs. Although AEC does not contribute substantially to routine MWF condition monitoring, it can be a useful tool for assessing the impact of non-biocidal MWF additives on MWF biostability.

\section{Acknowledgements}

The authors gratefully acknowledge the support of Blaser Swisslube, Hasle-Ruegsau, Switzerland, and LuminUltra Technologies, Ltd, Fredericton, New Brunswick, Canada for their support of this project. 


\section{Conflicts of Interest}

There are no conflicts of interest influencing the work reported in this paper.

\section{References}

1. (2019) Standard Classification for Metalworking Fluids and Related Materials, ASTM International, Pennsylvania, US.

2. Passman FJ (2018) Chapter 11-Microbiology of Metalworking Fluids. J Byers, Boca Raton (Eds), Metalworking Fluids p. 241-284.

3. Passman FJ (2008) Metalworking Fluid Microbes-What We Need to Know to Successfully Understand Cause and Effect Relationships. Tribol Trans 51(1): 107-117.

4. Passman FJ (2010) Current Trends in MWF Microbicides. Tribol Lub Technol 66(5): 31-38.

5. Passman FJ (2018) Chapter 11-Microbiology of Metalworking Fluids. J Byers, Boca Raton (Eds). Metalworking Fluids p. 241-284.

6. (2016) Standard Test Method for Measurement of Adenosine Triphosphate in Water-Miscible Metalworking Fluids, ASTM International, Pennsylvania, US.

7. Passman FJ, Egger G II, Hallahan S, Skinner B, Deschepper M (2009) Realtime Testing of Bioburdens in Metalworking Fluids Using Adenosine Triphosphate. Tribol Trans 52(6): 288-292.

8. Koch T, Passman F, Rabenstein A (2015) Comparative study of microbiological monitoring of water-miscible metalworking fluids. Int Biodeter \& Biodeg 98(1): 19-25.
9. Atkinson DE, Walton GM (1967) Adenosine Triphosphate Conservation in Metabolic Regulation. J Biol Chem 242(13): 3239-3241.

10. Wiebe WJ, Bancroft K (1975) Use of the Adenylate Energy Charge Ratio to Measure Growth State of Natural Microbial Communities. Proc Nat Acad Sci 72(6): 2122-2115.

11. Thomas KC, Dawson PSS (1977) Variations in the Adenylate Energy Charge During Phased Growth (Cell Cycle) of Candida utilis Under Energy Excess and Energy-Limiting Growth Conditions. J Bacteriol 132(1): 36-43.

12. Karl DM (1980) Cellular Nucleotide Measurements and Applications in Microbial Ecology. Microbiol Rev 44(4): 739-796.

13. Passman FJ, Küenzi P (2015) A Differential Adenosine Triphosphate Test Method for Differentiating between Bacterial and Fungal Contamination in Water-Miscible Metalworking Fluids. Int Biodeter Biodeg 99(1): 125137.

14. Li L, Mendis N, Trigui H, Oliver JD, Faucher SP (2014) The Importance of Viable but Non-Culturable State in Human Bacterial Pathogens. Frontiers Microbiol 5: 1-20. 